\title{
ASSESSMENT OF POSTOPERATIVE MANAGEMENT IN PATIENT WITH OSA AFTER TONSILLECTOMY
}

García Fernández E., Ríos Llorente A., Ruíz Chirosa M.C., Pascual Lorenzo M.J., Rodríguez Rojo M., Morales C.

\section{CHILHOOD OSA 5.7\%. Main cause: TONSILLAR HYPERTROPHY. PACU or ICU for these patients?}

\section{How many of these children have a previus POLISOMNOGRAPHY?}

\section{MATERIAL AND METHODS:}

Descriptive cross-sectional study in 56 patients undergoing tonsillectomy during the year 2014. We exclude patients older than 14 years. The program used was SPSS 20.0.

\section{RESULTS:}

OSA Incidence

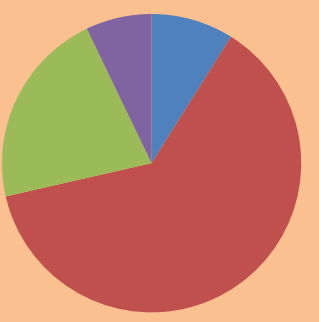

Previous Polisomnography $\mathbf{3 7 . 5 \% .}$

All patients with complications had OSA.

Moderate-mild OSA with complications: $34.28 \%$

Severe OSA with complications: $41.66 \%$

\begin{tabular}{|l|l|}
\hline Postoperative complications & N (\%) \\
\hline Nausea and vomiting & $2(3.6 \%)$ \\
\hline Desaturation & $3(5.4 \%)$ \\
\hline Airway obstruction & $2(3.6 \%)$ \\
\hline Bronchospasm & $1(1.8 \%)$ \\
\hline Laryngospasm & $1(1.8 \%)$ \\
\hline Postintubation laryngitis & $1(1.8 \%)$ \\
\hline Odynophagia & $3(5.4 \%)$ \\
\hline Postoperative agitation & $15(26.8 \%)$ \\
\hline Hemorrhage & $2(3.6 \%)$ \\
\hline Reintubation & $2(3.6 \%)$ \\
\hline Others & $1(1.8 \%)$ \\
\hline
\end{tabular}

27 patients (48.21\%) had one or more risk factors for postoperative complications. Of this group 14 (51\%) had some complication and all had OSA. ICU admission is significantly associated with risk of postoperative complications $(p<0.05)$.

\section{CONCLUSIONS}

$-<3$ years old $(\mathrm{P}<0.05)$ having complications after surgery $\rightarrow$ BETTER SELECTION OF PATIENTS

-Many OSA patients are classified without a polisomnography.

-Patient with OSA+risk factor= Complication after tonsillectomy $\rightarrow$ Postoperative care in ICU, not PACU 